\title{
The Complex Model Of The Quantum Universe
}

\author{
Dr. Narayan Kumar Bhadra \\ L. S. S. S. High School(H.S) 24 Parganas(N), West Bengal, India
}

\begin{abstract}
We study the line element proposed by the quantum vacuum Kantowshi-Sachs universe without cosmological constant. We consider the scale factor $b\left(=i R_{I}\right)$ of the metric to be imaginary and the other $a(=R)$ is real, of the Kantowski-Sachs quantum state space-time. It is seen, that in the Einstein's space-time, the numerical volume of the matter universe once been squeezed in a zero volume, we may assume then the matter universe transferred into another phase by the phase transition system with the help of latent energy group $S U(6)[S U(11) \supset S U(5) \times S U(6) \times U(1)]$ The subgroup $S U(6)$ has been interpreted as a new type of energy source other than $S U(5)[S U(5) \supset$ $S U(3) \times S U(2) \times U(1)$, where $S U(3)$ the strong energy group, $S U(2)$ the weak energy group \& $U(1)$ the electro dynamics]. We suppose that the original phase transition takes place before the GUT phase transition i.e. in the stage of SUT (Super Unified Theory) phase transition. According to the classical theory it may be assumed an imaginary scale factor, that means, the universe is then belongs to the pseudo-tachyon-field. It may be compared with the internal space ' $a$ ' of the extradimension ' $D$ ' expressed by the Kaluza-Klein cosmological universe.

Thus we think that the universe is numerically as a complex space-time $R+i R_{I}$ rather than it was measured as only the real part classically. Solving the metric tensors and getting the energy tensors $\mathrm{T}_{\mathrm{o}}^{\mathrm{o}}=\mathrm{T}_{1}^{1}$ and $\mathrm{T}_{2}^{2}=\mathrm{T}_{3}^{3}$ in the $10(=4+6)$-dimensional space-time under the exchange $\mathrm{R}_{\mathrm{I}} \leftrightarrow \mathrm{R}$ Hence we introduce a wider universe, other than Einstein's universe. We derived Einstein universe from wider universe.

We also study the Chaplygin gas Friedmann-Robertson-Walker quantum cosmological models in the presence of negative cosmological constant. We consider a $(4+D)-$ dimensional Friedmann-Robertsion-Walker type Universe having complex scale factor $R+i R_{I}$ We apply the Schutz's variational formalism to recover the notion of time and this give rise to Wheeler-DeWitt equations for the scale factor $R$, corresponding to 4 -dimensional universe and as well as $R_{I}$ for $D$ dimensional space. By introducing an exotic matter in the form of perfect fluid with an special equation of state, as the space-time part of the higher dimensional energy-momentum tensor, a four dimensional effective decaying cosmological term appears as $\lambda \sim R^{-m}$ with $0 \leq m \leq 2$, playing the role of an evolving dark energy in the universe. By taking $m=2$, which has some interesting implications in the reconciling observations with inflationary models and is consistent with quantum tunneling the resulting Einstein's field equations yield the exponential solutions for the scale factors $R_{I}$ and $R$. We use the eigen functions in order to construct the wave packets and obtain the time-dependent expectation value of the scale factors. Since the expectation value of the scale factors never tend to the singular point, we have an initial indication that this model may not have singularities at the quantum level.

Again we study the Hartle-Hawking no boundary proposal in which wave function of the universe is given by a path integral over all compact Euclidean 4-dimensional geometries and mater fields that have the 3-dimensional argument of the wave function on their one and only boundary. We suppose that the original phase transition takes place before the GUT phase transition i.e. in the stage of SUT (Super Unified Theory) phase transition. After the phase transition, there is a fluctuations of the scalar field $\phi$, over a smooth average value. These fluctuations result in fluctuations of energy density. In this paper, we choose the potential $V(\phi)$ as a exponential function of time " $t$ " and consider a complex radius of the Friedmann-Robertson-Walker model.
\end{abstract}

\section{Introduction:}

There is no consensus yet on how the universe initially came to be, the general assumption is that perhaps an energetic fluctuation caused the universe to tunnel into existence from quantum foam. The spontaneous symmetry breaking of the unified field occurred, thereby separating gravity, matter fields and GUT force field, as well as initiating the expansion of the universe. 
In 1915 Einstein published the general theory of relativity. He expected the universe to be 'closed' and to be filled with matter. Again, if we go out-side the gravitating sphere, we see the gravitation would be weaker and weaker. According to Einstein's general relativity, the matter-space-time cannot be separated by any cost. Thus, out-side the Einstein's universe, where real time can not be defined, so the corresponding space (although, the matter belongs to another phase) must be measured as imaginary. Thus the space-time of the universe is actually a complex space-time. Here we consider the real space-time (i.e. unfolded) for Einstein and imaginary space-time (i.e. folded) for us. We found a relation between folded and unfolded space-time of the universe by using Wheeler De-Witt equation. The generalized solution for the Einstein field equations for a homogeneous universe was first presented by Alexander Friedmann. The Friedmann equation for the evolution of the cosmic scale factor $\mathrm{R}(\mathrm{t})$ which represents the size of the universe, is

$$
\left[\frac{R(t)}{R(t)}\right]^{2}=\frac{8 \pi G}{3} \rho(t)-\frac{k c^{2}}{R^{2}(t)} \text {. i.e. } R^{2}(t)=\frac{8 \pi G}{3}\left[\rho(t) \cdot R^{3}(t)\right] \frac{1}{R(t)}-k c^{2}
$$

Differentiating the above equation with respect to time ' $t$ ', and since the total matter in a given expanding volume is unchanged, i.e. $\rho(t) \cdot R^{3}(t)$ is constant. We have,

$$
2 \dot{R}(t) \ddot{R}(t)=-\frac{8 \pi G}{3} \rho(t) R^{3}(t) \frac{\dot{R}(t)}{R^{2}(t)} \quad \text { i.e } \quad \frac{\ddot{R}(t)}{R(t)}=-\frac{4 \pi G}{3} \rho(t)
$$

Since $\mathbf{R}$ is always negative, at a finite time in the past $\mathbf{R}$ must have been equal to zero. Then, according to these models, the contents of all the galaxies must have once been squeezed together in a small volume where the temperature would have been immensely high. The radiation left over from this fireball must still be around today, although cooled to a much lower temperature due to expansion of the universe. So as the size ' $R$ ' of the Einstein matter universe numerically squeezed in a zero volume, then we may assume the size of the Einstein's matter universe become $i_{I}$ in other phase which can be measured classically, as the energy never die at all.

So we may consider the scale factor ' $R$ ' of the Einstein universe is only the real part and there may exist an imaginary part (pseudo-space) ' $i \mathrm{R}_{\mathrm{I}}$ ' and hence the size of the universe is actually may be written in the form $R+i R_{I}$.

If we consider the $(4+\mathrm{D})$-dimensional Kaluza-Klein cosmology with a Robertson-Walker type metric having two scale factors ' $a$ ' and ' $R$ ', corresponding to $D$-dimensional internal space and 4-dimensional space, respectively. In the expansion of ' $R$ '-universe, the internal space ' $a$ ' decreases as the space $R$ increases. Avoiding ' $R$ '-universe squeezed in a zero volume, then we may assume that the matter universe must be changed into another phase by the phase transition method with the help of latent energy group SU(6).

It is then compared with the D-dimensional internal space ' $a$ ' $\left(=i R_{I}\right)$ of the Kaluza-Klein cosmology, with the Einstein's 4-dimensional space of the scale factor R. The knowledge about the quantum state space for the gravity system and gravity matter system are very limited and the definition of the inner-product in quantum state space has not been found.

A natural query is what happened before unification-may be called super unified field. The vacuum universe $\mathrm{U}(11)$ is thermodynamically equilibrium with the infinite boundary $\left(\mathrm{R} \rightarrow \propto, \mathrm{R}_{\mathrm{I}} \rightarrow \propto\right.$ ) like a plain white paper. The break-down of the special unitary group $\mathrm{SU}(11)$ of $\mathrm{U}(11)$ into $\mathrm{SU}(6) \times$ $\mathrm{SU}(5) \times \mathrm{U}(1)$, under the pre-distribution of energy when it is reached below the "critical point" (it is compared with the curie point of the magnet).

The breakdown of SUT symmetry group SU(11), gave two fundamental group like SU(6) $\times$ $\mathrm{SU}(5)$ leads to a phase transition and then the fundamental group $\mathrm{SU}(5)$ breaks into subgroup like $\mathrm{SU}(3) \times \mathrm{SU}(2)_{\mathrm{L}} \times \mathrm{U}(1)$, in which the scalar field $\phi$ changes. The original vacuum, with false vacuum $(\phi=0)$ is no longer the true vacuum $(\phi=\sigma)$. The inflationary stage arises, however, if the true vacuum is not immediately attained.

After the separation of two type energies $\mathrm{SU}(5) \& \mathrm{SU}(6)$, they want to interact each other and has a tendency to unify once again, as a result the direction of the energy group SU(6) is opposite to the direction of the energy group SU(5), remaining the temperature unaltered and then an inflation occurred instantaneously. An analogy will illustrate the scenario. Suppose steam is being cooled through the phase transition temperature of $100^{\circ} \mathrm{c}$. Normally we expect the steam to condense to water at this temperature. However, it is possible to super-cool the steam to 
temperature below $100^{\circ} \mathrm{c}$, although it is then in an unstable state. The instability set in when certain parts of the steam condense to droplets of water which then coalesce and eventually the condensation is complete. In the super-cooled state the steam still remains its latent heat, which is released as the droplets form.

It is very convenient to construct a quantum perfect fluid model. Schutz's formalism gives dynamics to the fluid degrees of freedom in interaction with the gravitational field. Using a proper canonical transformations, at least one conjugate momentum operator associated with matter appears linearly in the action integral. Therefore, a Schrodinger-like equation can be obtained where the matter plays the role of time. Moreover, recently, some applications of the Schutz's formalism have been discussed in the frame work of the perfect fluid Stephani Universe and Friedmann-Robertson-Walker Universe in the presence of Chaplygin gas.

\section{Intelligence: $\mathrm{SU}(6)$}

In the transformations under the group SU(6), the basic fields here are the latent energy field and we have $\quad \mathrm{U}=\exp (-\mathrm{iH}) \ldots \ldots \ldots .(1)$. Where $\mathrm{H}$ is a $6 \times 6$ Hermitian matrix of zero trace. We have 35 matrix charges $I_{1}, I_{2}, I_{3}, \ldots \ldots \ldots . I_{35}$ out of which five matrices are diagonal. corresponding to this, we have 35 bosons. For want of any specific designation, they are referred to simply as $\mathrm{J}_{\mathrm{k}}$. There were no change takes place for exchanging the bosons namely $\mathrm{J}_{\mathrm{k} 3}, \mathrm{~J}_{\mathrm{k} 8}, \mathrm{~J}_{\mathrm{k} 15}, \mathrm{~J}_{\mathrm{k} 24}$, $\mathrm{J}_{\mathrm{k} 35}$, corresponding to the said five diagonal matrices. We expect the participating interactions of the bosons $\mathrm{J}_{\mathrm{k}}$ to have comparable strength. The $\mathrm{J}_{\mathrm{k}}$ bosons are expected to generate a latent force. This force is believed to be potentially so large that the exotic matter fluid are expected to transfer into the ordinary matter and then everything of the universe.

As the energy group SU(5) advanced for unification with SU(6), the strength of weak force gradually increases and the strength of strong force decreases, ultimately the unification occurred at the extreme situation.

\section{Super Unified Theory}

It can be expected, that for the symmetry breaking of SU(11), created an amount of positive energy, negative energy and an equivalent amount of latent energy.

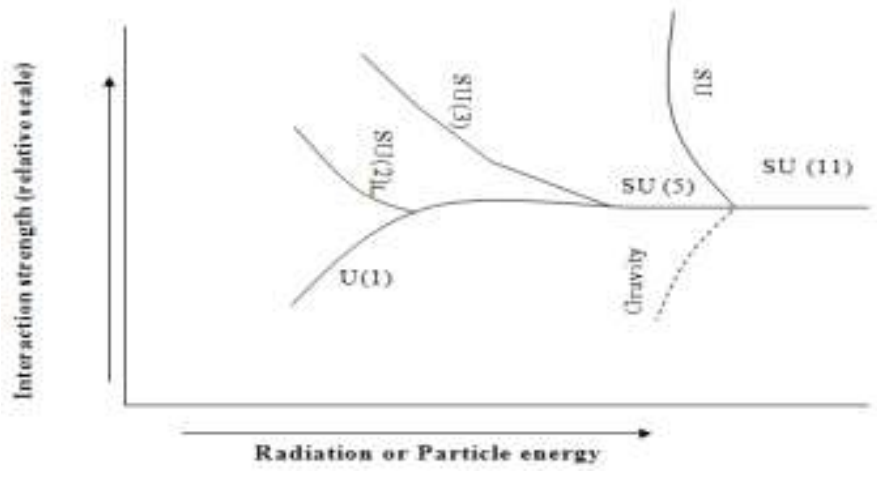

\section{Einstein's solution in general phase without cosmological constant:}

Imagine, a uniform distribution of matter filling the infinite Euclidean space in a phase other than Einstein's universe. We know that any finite distribution of pressure-free matter would tend to shrink under its own gravity.

We consider the closed surface from the very early universe as rectangular-parallelepiped after then a cubical box and then spherical space-time. The equation of the diagonal of the parallelepiped as

$$
\mathrm{x}_{1}^{2}+\mathrm{x}_{2}^{2}+\mathrm{x}_{3}^{2}=\mathrm{r}_{2}^{2}
$$

Consider the side of the cubical box is $\mathrm{r}_{1}$ and also consider the diagonal remains unchanged for parallelepiped and cubical box.

Hence the diagonal of the cubical box is

$$
r_{2}=\sqrt{3} r_{1}
$$

Now we consider the equation (3) of a 3-surface in Cartesian co-ordinates $\mathrm{x}_{1}, \mathrm{x}_{2}, \mathrm{x}_{3}, \& \mathrm{x}_{4}$ by

$$
\mathrm{x}_{1}^{2}+\mathrm{x}_{2}^{2}+\mathrm{x}_{3}^{2}+\mathrm{x}_{4}^{2}=\mathrm{S}_{1}^{2}
$$


Where $S_{1}$ is the radius of the 3 -surface of a four dimensional hyper-sphere. We consider the total mater in the cubical surface and Einstein's spherical surface remains unaltered. Hence

$$
\mathrm{r}_{1}^{3} \varepsilon_{1}=\frac{4}{3} \pi \mathrm{R}^{3} \varepsilon \quad \text { i.e. } \quad \mathbf{r}_{1}=\left(\frac{4 \pi}{3}\right)^{1 / 3}\left(\frac{\varepsilon}{\varepsilon_{1}}\right)^{1 / 3} \mathbf{R}
$$

[Taking only the real part]

$$
\therefore \quad r_{2}=\sqrt{3} r_{1}=\sqrt{3}\left[\frac{4 \pi}{3}\right]^{1 / 3}\left[\frac{\varepsilon}{\varepsilon_{1}}\right]^{1 / 3} R
$$

Interms of a constant negative curvature, the equation (4) becomes

$$
\text { Here, } \mathbf{S}_{\mathbf{1}}=\mathbf{r}_{\mathbf{2}}=\phi(\mathbf{t}) \cdot \mathbf{R}(\mathbf{t}) \quad \text { Where } \quad \phi(\mathbf{t})=\sqrt{\mathbf{3}}\left(\frac{\mathbf{4 \pi}}{\mathbf{3}}\right)^{\mathbf{1 / 3}}\left(\frac{\varepsilon}{\varepsilon_{1}}\right)^{1 / 3}
$$

Where $\varepsilon, \varepsilon_{1}$ are the energy densities of the two phase respectively. Comparing with the Einstein's universe, the most general line element satisfying the Weyl-postulate and the cosmological principal is given by

$$
d s^{2}=c^{\prime 2} d t^{2}-S^{2}(t)\left[\frac{d r^{2}}{1-k r^{2}}+r^{2}\left(d \theta^{2}+\operatorname{Sin}^{2} \theta d \phi^{2}\right)\right]
$$

Where, $S(t)=\phi(t) . R(t)$

$$
|\phi(t)| \geq \sqrt{3}\left(\frac{4 \pi}{3}\right)^{1 / 3}
$$

And the Einstein's equations become,

$$
\begin{aligned}
& 2 \frac{\ddot{S}}{S}+\frac{\dot{S}^{2}+k c^{\prime 2}}{S^{2}}=\frac{8 \pi G}{c^{\prime 2}} T_{1}^{1}=\frac{8 \pi G}{c^{\prime 2}} T_{2}^{2}=\frac{8 \pi G}{c^{\prime 2}} T_{3}^{3} \\
& \& \quad \frac{S^{2}+k c^{\prime 2}}{S^{2}}=\frac{8 \pi G}{3 c^{\prime 2}} T_{o}^{0} \\
& S=\dot{\phi}(t) R(t)+\dot{R}(t) \phi(t)
\end{aligned}
$$

$$
\ddot{S}=\ddot{\phi}(\mathbf{t}) \mathbf{R}(\mathbf{t})+2 \mathbf{R}(\mathbf{t}) \phi(t)+\mathbf{R}(\mathbf{t}) \phi(\mathbf{t})
$$

\section{Einstein Universe derived from wider universe:}

From the equation (10) \& (11) we have the relation,

$$
\frac{d}{d s}\left(\varepsilon_{1} S^{3}\right)+3 p^{\prime} S^{2}=0
$$

when $\quad \mathrm{T}_{1}^{1}=\mathrm{T}_{2}^{2}=\mathrm{T}_{3}^{3}=-\mathrm{p}^{\prime} \quad$ and $\quad \mathrm{T}_{\mathrm{o}}^{\mathrm{o}}=\varepsilon_{1}$

At the very early epoch, $\mathrm{p}^{\prime}=0$, then

$$
\varepsilon_{1} S^{3}=\text { constant. }
$$

By the equation (12) we have $\varepsilon_{1} \phi^{3}(\mathrm{t}) \cdot \mathrm{R}^{3}(\mathrm{t})=\mathrm{constan} \mathrm{t}$

Consider, when $\mathrm{R}=\mathrm{R}_{\mathrm{o}}, \varepsilon=\varepsilon_{\mathrm{o}}$ then

$$
\text { i.e. } \quad \varepsilon \mathrm{R}^{3}=\text { constant. [ by the equation (8)] }
$$

$$
\varepsilon \mathrm{R}^{3}=\varepsilon_{\mathrm{o}} \mathrm{R}_{\mathrm{o}}^{3}
$$

Which may be found by the Einstein's universe at the early epoch when pressure $p=0$, by using the Friedmann equations and with the help of Robertson-Walker line element. 


$$
d s^{2}=c^{2} d t^{2}-R^{2}(t)\left[\frac{d r^{2}}{1-k r^{2}}+r^{2}\left(d \theta^{2}+\operatorname{Sin}^{2} \theta d \phi^{2}\right)\right]
$$

Again in absence of any external forces, the velocity $u^{i}$ satisfies the geodesic equation

$$
\frac{\mathrm{du}^{\mathrm{i}}}{\mathrm{ds}}+\Gamma_{\mathrm{K} \ell}^{\mathrm{i}} \mathrm{u}^{\mathrm{K}} \mathrm{u}_{\ell}=0
$$

Substitute $\Gamma_{\mathrm{K} \ell}^{\mathrm{i}}$ in the equation (9) gives the result

$$
\mathrm{u}^{\mathrm{\mu}} \mathrm{S}^{2}=\text { constant. }
$$

However, $\mathrm{u}^{\mu}$ measures the velocity in the co-moving $(\mathrm{r}, \theta, \phi)$ co-ordinates, we have

$$
u^{\mu} \phi^{2}(t) R^{2}(t)=\text { constant } \ldots .
$$

Again, if we substitute $\Gamma_{\mathrm{kl}}^{\mathrm{i}}$ in the equation (16) which gives the result

$u^{\mu} R^{2}(t)=$ constant

Now, if we compare (20) with (19), we get $\phi(t)=$ constant.

$$
\text { i.e. } \quad \varepsilon_{1}(\mathrm{t}) \propto \varepsilon(\mathrm{t})
$$

Which indicates the large or small matter energy density in the vapor phase (so called nothing) changes to the large or small matter energy density in the liquid phase (Einstein's Universe) and hence it is found, an important fact, that the existence of discrete structure in the universe, ranging from galaxies to super-clusters.

\section{V. $\quad$ Einstein field equation in complex quantum state :}

The work covered in the Einstein field equations did not tell us the important item of information about the universe is what happened, when the volume of the matter universe squeezed into zero volume and there before. To find the answer to this question it is necessary to do beyond the concept of Einstein universe. We need a new concept with the Einstein's universe to proceed any further, and Einstein's general relativity with complex space-time is one of such theory. We will consider alternative approaches to cosmology but for the present is Kantowski-Sachs universe. We have the line element to start with:

$$
\mathrm{ds}^{2}=-\mathrm{N}^{2}(\mathrm{t}) \mathrm{dt}^{2}+\mathrm{a}^{2}(\mathrm{t}) \mathrm{dr}^{2}+\mathrm{b}^{2}(\mathrm{t})\left(\mathrm{d} \theta^{2}+\operatorname{Sin}^{2} \theta \mathrm{d} \phi^{2}\right)
$$

The only nontrivial Einstein equations of the above metric (with $\mathrm{N}=1$ are)

$$
\begin{gathered}
\frac{\mathbf{b}^{2}}{\mathbf{b}^{2}}+\frac{2 \mathbf{a} \dot{b}}{a b}+\frac{1}{b^{2}}=-\frac{8 \pi G}{c^{/ 4}} T_{0}^{0} \\
\frac{2 b}{b}+\frac{b^{2}}{b^{2}}+\frac{1}{b^{2}}=-\frac{8 \pi G}{c^{/ 4}} T_{1}^{1} \\
\frac{\ddot{a}}{a}+\frac{\ddot{b}}{b}+\frac{a \dot{b}}{a b}=-\frac{8 \pi G}{c^{/ 4}} T_{2}^{2}=-\frac{8 \pi G}{c^{/ 4}} T_{3}^{3}
\end{gathered}
$$

Where $c^{\prime}$ is the velocity of photon-like particle in vapor stage and $c^{\prime}>c$, the velocity of photon. We next consider $a=R \& b=i R_{I}$, [where $i=\sqrt{-1} \quad$ ]. Then the equation (23), (24) \& (25) becomes

$$
\begin{aligned}
& \frac{\dot{\mathbf{R}}_{\mathrm{I}}^{2}}{\mathbf{R}_{\mathrm{I}}^{2}}+\frac{2 \dot{\mathbf{R}}_{\mathrm{R}}}{\mathbf{R R}_{\mathrm{I}}}-\frac{1}{\mathbf{R}_{\mathrm{I}}^{2}}=-\frac{8 \pi \mathrm{G}}{\mathrm{c}^{14}} \mathbf{T}_{0}^{0} \\
& \frac{2 \ddot{\mathbf{R}}_{\mathrm{I}}}{\mathbf{R}_{\mathrm{I}}}+\frac{\dot{\mathbf{R}}_{\mathrm{I}}^{2}}{\mathbf{R}_{\mathrm{I}}^{2}}-\frac{1}{\mathbf{R}_{\mathrm{I}}^{2}}=-\frac{8 \pi \mathrm{G}}{\mathrm{c}^{/ 4}} \mathbf{T}_{1}^{1}
\end{aligned}
$$

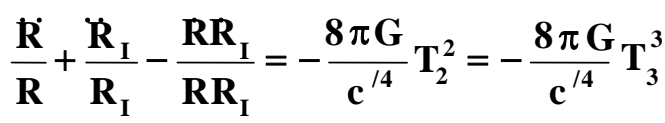


Before we consider specific forms of $\mathrm{T}_{\mathrm{k}}^{\mathrm{i}}$, it is worth noting that three properties must be satisfied by the energy tensor in the present framework of cosmology. The first is obviously define negative pressure by $\mathbf{T}_{2}^{2}=\mathbf{T}_{3}^{3}$ The second $\mathrm{T}_{\mathrm{o}}^{\mathrm{o}}$, is

define the matter energy density and the third $\mathrm{T}_{1}^{1}$ is define the latent energy density. If we take

$$
\mathbf{R}_{\mathrm{I}}=\square_{\mathbf{p}} \mathbf{e}^{\mathbf{H}_{\mathrm{I}} \mathbf{t}} \quad \& \quad \mathbf{R}=\square_{\mathbf{p}} \mathbf{e}^{\mathbf{H}_{\mathbf{R}} \mathbf{t}}
$$

Also we have from (26), (27) \& (28), we get

$$
T_{0}^{0}=\frac{c^{/ 4}}{8 \pi G}\left[\frac{e^{-2 H_{I} t}}{\ell_{p}^{2}}-H_{I}^{2}-2 H_{I} H_{R}\right]
$$

$$
\& \quad \mathbf{T}_{2}^{2}=\mathbf{T}_{3}^{3}=-\frac{\mathrm{c}^{14}}{8 \pi \mathrm{G}}\left[\mathrm{H}_{\mathrm{R}}^{2}+\mathrm{H}_{\mathrm{I}}^{2}+\mathrm{H}_{\mathrm{R}} \mathrm{H}_{\mathrm{I}}\right] \quad \mathrm{T}_{1}^{1}=\frac{\mathrm{c}^{/ 4}}{8 \pi \mathrm{G}}\left[\frac{\mathrm{e}^{-2 \mathrm{H}_{\mathrm{I}}}}{\ell_{\mathrm{p}}^{2}}-3 \mathrm{H}_{\mathrm{I}}^{2}\right]
$$

Now, it is clear that, if $H_{R}=H_{I}$ i.e. $R=R_{I}$. Which is possible for $6+4=10$ dimensional space-time. Then the equations (29) \& (31) are identical

$$
\begin{aligned}
& \text { i.e } \quad \mathbf{T}_{0}^{0}=\mathbf{T}_{1}^{1}=-\frac{3 \mathbf{c}^{/ 4}}{8 \pi G}\left[\mathbf{H}_{\mathrm{R}}^{2}-\frac{\mathrm{e}^{-2 \mathrm{H}_{\mathrm{R}}^{\mathrm{t}}}}{3_{\ell_{\mathrm{p}}^{2}}^{2}}\right] \\
& T_{2}^{2}=T_{3}^{3}=-\frac{3 c^{/ 4}}{8 \pi G}\left[H_{R}^{2}\right]=-\frac{3 c^{/ 4}}{8 \pi G}\left[H_{I}^{2}\right]
\end{aligned}
$$

Now, if $T_{2}^{2}=T_{3}^{3}=0$ then, $H_{I}=H_{R}=0$ i.e. $R_{I}=$ constant. Hence there is a maximum of $R_{I}$

At $\quad \mathrm{T}_{\mathrm{o}}^{\mathrm{o}}=\mathrm{T}_{1}^{1}=0 \quad$ we have, $\quad \mathbf{t}=\frac{\mathbf{1}}{\mathbf{H}_{\mathbf{R}}} \log \left[\frac{\mathbf{1}}{\sqrt{\mathbf{3}} \cdot \ell_{\mathbf{p}} \mathbf{H}_{\mathbf{R}}}\right]$
which is not possible, i.e. $\mathrm{T}_{\mathrm{o}}^{\mathrm{o}}=\mathrm{T}_{1}^{1} \neq .0$

For derivation it is convenient to write

and

$$
T_{o}^{0}=T_{1}^{1}=\varepsilon=-\frac{3 c^{/ 4}}{8 \pi G}\left[H_{R}^{2}-\frac{1}{3 \ell_{p}^{2}} e^{-2 H_{R} t}\right]
$$

It relates the pressure p to the energy density $\varepsilon$. Hence from equations (35) \& (36) we have

$$
\varepsilon+p=\frac{1}{8 \pi G_{\ell p^{2}}} e^{-2 H_{R} t}\left[\text { consider, } c^{\prime}=1\right]
$$

\section{Behaviour of Entropy in the Complex Space-Time}

The second laws of thermodynamics tell us that the entropy in a given volume $S^{3}$ stays constant as the volume expands adiabatically.

That means $\quad \mathrm{p}+\varepsilon=\frac{\sigma \mathrm{T}}{\mathrm{S}^{3}}$, where $\sigma$ is a constant is and $\mathrm{T}$ is the temperature. Where $\mathrm{S}$ is the
scale factor, Hence,

Separating real and imaginary part, we get

$$
\varepsilon+\mathrm{p}=-\frac{\sigma \mathrm{T}}{4 \mathrm{R}^{3}}
$$

Hence from equations (37) \& (39) we have

i.e $\quad \mathrm{T}=-\frac{\ell_{\mathrm{p}}}{2 \pi \sigma \mathrm{G}} \mathrm{e}^{\mathrm{H}_{\mathrm{R}} \mathrm{t}}$

$$
-\frac{\sigma \mathrm{T}}{4 \mathrm{R}^{3}}=\frac{\mathrm{e}^{-2 \mathrm{H}_{\mathrm{R}} \mathrm{t}}}{8 \pi \mathrm{G} \ell \mathrm{p}^{2}}
$$

We take $\mathrm{T}=\mathrm{T}_{\mathrm{o}}$ (say) when $\mathrm{t}=0$,

Then

$$
\mathrm{T}=\mathrm{T}_{\mathrm{o}} \mathrm{e}^{\mathrm{H}_{\mathrm{R}} \mathrm{t}}
$$

Which is the temperature of the universe in the super gravity stage, when the wave function is symmetric under the exchange $\mathrm{R} \leftrightarrow \mathrm{R}_{\mathrm{I}}$

One may assume a sudden drop of temperature due to the separation of latent energy in the phase transition, which is not measurable by any physical instrument. 


\section{Cosmology of perfect fluid and Chaplygin gas model}

We study the metric considered in which the space time is assumed to be of FriedmannRobertson-walker type,

$$
\mathrm{ds}^{2}=-\mathrm{N}^{2}(\mathrm{t}) \mathrm{dt}^{2}+\mathrm{a}^{2}(\mathrm{t}) \mathrm{h}_{\ell \mathrm{m}} \mathrm{dx} \mathrm{x}^{\ell} \mathrm{dx}
$$

where $\mathrm{N}(\mathrm{t})$ is the lapse function, $\mathrm{a}(\mathrm{t})$ is the scale factor of the space time, $\mathrm{h}_{\ell \mathrm{m}}$ is the metric. The metric(41) can be inserted in the Einstein Hilbert action plus a generalized Chaplygin gas in the formalism developed by Schutz and we obtain

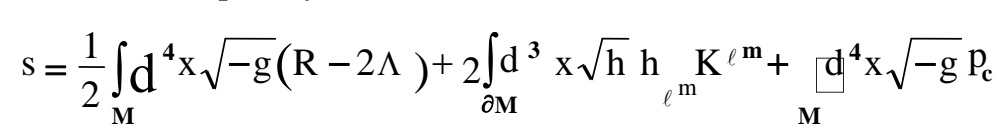

where $\mathrm{g}_{\mu v}$ is the metric, $\mathrm{R}$ is the Ricci scalar, $\mathrm{K}^{\ell \mathrm{m}}$ is the extrinsic curvature, $\Lambda$ is the cosmological constant, and $\mathrm{h}_{\ell \mathrm{m}}$ is the induced metric over the three-dimensional spatial hypersurface, which is the boundary $\partial \mathbf{M}$ of the 4-dimensional manifold $\mathrm{M}$. We choose units such that the factor $8 \pi \mathrm{G}$ becomes equal to one, $\mathrm{p}_{\mathrm{e}}$ denotes the Chaplygin gas pressure. The last term of (42) represents the matter contribution to the total action.

We assume the energy-momentum tensor $\mathrm{T}_{\mu, v}$ of space time to be an exotic $\chi$ fluid with the equation of state

$$
\mathbf{p}_{\chi}=\left(\frac{\mathbf{m}}{\mathbf{3}}-\mathbf{1}\right) \rho_{\chi}=\alpha \rho_{\chi}
$$

$$
\text { [where } \quad \alpha=\frac{m}{3}-1 \text { ] }
$$

Where $\mathrm{p}_{\chi}$ and $\rho_{\chi}$ are the pressure and density of the perfect fluid, respectively and the parameter $\mathrm{m}$ is restricted to the range $0 \leq \mathrm{m} \leq 2$ when $\mathrm{m}=2$, then $\alpha=1 / 3$. It is worth nothing that the equation of state (43) with $0 \leq \mathrm{m} \leq 2$ resembles a universe with negative pressure, violation the strong energy condition and this violation is required for universe to be accelerated.

From thermo-dynamical considerations and using the constraints for the fluid, if we drop the surface term in the action (42), and use the canonical transformation then the Super Hamiltonian simplifies to

$$
\mathrm{H}=-\frac{\mathrm{p}_{\mathrm{a}}^{2}}{12 \mathrm{a}}+\Lambda \mathrm{a}^{3}-3 \mathrm{Ka}+\left[\mathrm{sp}_{\phi}^{1+\alpha}+\mathrm{Aa}^{3(1+\alpha)}\right]^{\frac{1}{1+\alpha}}
$$

Now, we study the Chaplygin gas expression in early and late times limits, namely for small scale factors

$\mathrm{Sp}_{\phi}^{1+\alpha}>>\mathrm{A} \mathrm{a}^{3(1+\alpha)}$ and large scale factors $\mathrm{Sp}_{\phi}^{1+\alpha}<<\mathrm{A} \mathrm{a}^{3(1+\alpha)}$, separately. For the early universe, we use the following expression

$$
\left(\left(\mathrm{Sp}_{\phi}^{1+\alpha}+\mathrm{Aa}^{3(1+\alpha)}\right)^{\frac{1}{1+\alpha}} \simeq \mathrm{S}^{\frac{1}{1+\alpha}} \mathrm{p}_{\phi}\left[1+\frac{1}{1+\alpha} \frac{\mathrm{Aa}^{3(1+\alpha)}}{\mathrm{Sp}_{\phi}^{1+\alpha}}+\cdot\right] \ldots\right.
$$

Hence, up to the leading order, the super Hamiltonian takes the form

$$
\mathrm{H}=-\frac{\mathrm{p}_{\mathrm{a}}^{2}}{12 \mathrm{a}}+\Lambda \mathrm{a}^{3}-3 \mathrm{Ka}+\mathrm{S}^{\frac{1}{1+\alpha}} \mathrm{p}_{\phi}
$$

Again, we use canonical transformation, the super-Hamiltonian simplifies to

where $\quad \mathrm{p}_{\mathrm{T}}=\mathrm{S}^{\frac{1}{1+\alpha}} \mathrm{p}_{\phi}$

$$
\mathrm{H}=-\frac{\mathrm{p}_{\mathrm{a}}^{2}}{12 \mathrm{a}}+\Lambda \mathrm{a}^{3}-3 \mathrm{Ka}+\mathrm{p}_{\mathrm{T}}
$$

Where the momentum $\mathrm{p}_{\mathrm{T}}$, is the only remaining canonical variable associated with matter and appears linearly in the super-Hamiltonian. The parameter $\mathrm{K}$ defines the curvature of the spatial section, taking the values $0,1,-1$ for a flat, close or open universe, respectively. 
It is apparent that, up to the leading order, the Chaplygin gas plays the role of the dust fluid in the early time regime.

\section{Wheeler De-Witt equation}

An appropriate quantum mechanical description of the universe was introduce and developed by De-Witt. In quantum cosmology the universe, as a whole, is treated quantum mechanically and is described by a single wave function.

$\Psi\left(\mathrm{h}_{\mathrm{ij}}, \phi\right)$, defined on a manifold (super-space) of all possible three geometries and all matter field configurations. The wave function $\Psi\left(\mathrm{h}_{\mathrm{ij}}, \phi\right)$, has no explicit time dependence due to the fact that there is no real time parameter external to the universe. Therefore there is no Schrodinger wave equation but the operator version of the Hamiltonian constraint of the Dirac canonical quantization procedure, namely vanishing of the variation of the Einstein-Hilbert action $\mathrm{S}$ with respect to the arbitrary lapse function $\mathrm{N}$

$$
\mathrm{H}=\frac{\delta \mathrm{S}}{\delta \mathrm{N}}=0
$$

Which is written $\hat{\mathrm{H}} \Psi\left(\mathrm{h}_{\mathrm{ij}}, \phi\right)=0$

This equation is known as the Wheeler-DeWitt (WDW) equation. The goal of quantum cosmology by solving the WDW equation is to understand the origin and evolution of the universe, quantum mechanically. As a differential equation, the WDW Equation has an infinite number of solutions. To get a unique viable solution, we should also respect the question of boundary condition in quantum cosmology which is of prime importance in obtaining the relevant solutions for the WDW equation.

In principle, it is very difficult to solve the WDW equation in the super-space due to the large number of degrees of freedom of the of the gravitational and matter fields. This procedure is known as quantization in mini super-space, and will be used in the following discussion.

The Wheeler-Dewitt equation in mini-super-space can be obtained by imposing the standard quantization conditions
on the canonical momentum $\left[\mathrm{p}_{\mathrm{a}}=-\mathrm{i} \frac{\partial}{\partial \mathrm{a}}, \mathrm{p}_{\mathrm{T}}=-\mathrm{i} \frac{\partial}{\partial \mathrm{T}}\right]$ and requiring that the super Hamiltonian operator annihillates the wave function of the universe $(\hbar=1)$. Using $(46)$, we have

$$
\left[-\frac{\partial^{2}}{\partial \mathrm{a}^{2}}+\left(-12 \Lambda \mathrm{a}^{4}+36 \mathrm{Ka}^{2}\right)-\mathrm{i} 12 \mathrm{a} \frac{\partial}{\partial \mathrm{t}}\right] \Psi=0
$$

Here, $\mathrm{t}=\mathrm{T}$ corresponds to the time co-ordinate. Equation (47) takes the form of a Schrodinger equation $\quad i \frac{\partial \psi}{\partial t}=\hat{H} \psi$

Demanding that the Hamiltonian operator $\hat{\mathrm{H}}$ to be self-adjoint, the inner product of any two wave functions $\phi$ and $\Psi$ must take form

$$
(\phi, \Psi)=\int_{0}^{\infty} \mathrm{a} \phi^{*} \Psi \mathrm{da}
$$

On the other hand, the wave functions should satisfy the following boundary conditions

Using the separation of variables

$$
\Psi(0, \mathrm{t})=0 \text { or }\left.\frac{\partial}{\partial \mathrm{a}} \Psi(\mathrm{a}, \mathrm{t})\right|_{\mathrm{a}=0}=0
$$

The equations (47) take the form,

$$
\Psi(\mathrm{a}, \mathrm{t})=\mathrm{e}^{\mathrm{iEt}} \Psi(\mathrm{a})
$$

$$
-\frac{\partial^{2} \Psi(\mathrm{a})}{\partial \mathrm{a}^{2}}+\left(36 \mathrm{Ka}^{2}-12 \Lambda \mathrm{a}^{4}\right) \Psi(\mathrm{a})=12 \mathrm{aE} \Psi(\mathrm{a})
$$

For the late times $\left(\mathrm{Sp}_{\phi}^{1+\alpha}<<\mathrm{Aa}^{3(1+\alpha)}\right)$, we have

$$
\left(\mathrm{Sp}_{\phi}^{1+\alpha}+\mathrm{Aa}^{3(1+\alpha)}\right)^{\frac{1}{1+\alpha}} \simeq \mathrm{A}^{\frac{1}{1+\alpha}} \mathrm{a}^{3}\left[1+\frac{1}{1+\alpha} \frac{\mathrm{Sp}_{\phi}^{1+\alpha}}{\mathrm{Aa}^{3(1+\alpha)}}+\ldots \ldots\right]
$$

So, up to the first order, the super Hamiltonian (44) takes the following form

$-\alpha$ 


$$
\mathrm{H}=-\frac{\mathrm{p}_{\mathrm{a}}^{2}}{12 \mathrm{a}}+\Lambda \mathrm{a}^{3}-3 \mathrm{Ka}+\mathrm{A}^{\frac{1}{1+\alpha}} \mathrm{a}^{3}+\frac{\mathrm{A}^{\overline{1+\alpha}}}{\alpha+1} \mathrm{a}^{-3 \alpha} \mathrm{Sp}_{\phi}^{1+\alpha}
$$

Now, using the additional canonical transformation, the super-Hamiltonian simplifies to

where, $\mathrm{p}_{\mathrm{T}}=\frac{\overline{\mathrm{A}}^{\frac{\alpha}{\alpha+1}}}{1+\alpha} \mathrm{Sp}_{\phi}^{1+\alpha}$

$$
\mathrm{H}=-\frac{\mathrm{p}_{\mathrm{a}}^{2}}{12 \mathrm{a}}+\Lambda \mathrm{a}^{3}-3 \mathrm{Ka}+\mathrm{A}^{\frac{1}{1+\alpha}} \mathrm{a}^{3}+\mathrm{a}^{-3 \alpha} \mathrm{p}_{\mathrm{T}}
$$

Using canonical form of moment, the SWDW equation in the late time can be written as

$$
\frac{\partial^{2} \Psi}{\partial \mathrm{a}^{2}}-\left(36 \mathrm{Ka}^{2}-12 \Lambda \mathrm{a}^{4}-12 \mathrm{~A}^{\frac{1}{1+\alpha}} \mathrm{a}^{4}\right) \Psi=\mathrm{i} 12 \mathrm{a}^{1-3 \alpha} \frac{\partial \Psi}{\partial \mathrm{t}}
$$

For this case, the inner-product of any two functions $\phi$ and $\psi$ takes the form

Using the separation of variables

$$
(\phi, \Psi)=\int_{0}^{\infty} a^{1-3 \alpha} \phi^{*} \Psi d a
$$

We can obtain the time-independent SWDW equation as,

$$
-\frac{\partial^{2} \psi}{\partial \mathrm{a}^{2}}+\left(36 \mathrm{ka}^{2}-12 \Lambda \mathrm{a}^{4}-12 \mathrm{~A}^{\frac{1}{1+\alpha}} \mathrm{a}^{4}\right) \psi=12 \mathrm{Ea}^{1-3 \alpha} \psi(\mathrm{a})
$$

Using (50)\& (57), we get, $\quad-12 \mathrm{~A}^{\frac{1}{1+\alpha}} \mathrm{a}^{4} \psi(\mathrm{a})+12 \mathrm{aE} \psi(\mathrm{a})=12 \mathrm{Ea}^{1-3 \alpha} \psi(\mathrm{a})$

i.e. $A^{3 / 2} a^{3}+a E-E=0 \quad[\because \psi(a) \neq 0, a \neq 0$ and take $\alpha=-1 / 3]$

We take $a=R+i R_{I}$

then $\quad\left[A^{3 / 2}\left(R^{3}-3 R R_{I}^{2}\right)+E(R-1)\right]+i\left[A^{3 / 2}\left(\beta R^{2} R_{I}-R_{I}^{3}\right)+E R_{I}\right]=0$

Separating real and imaginary part, we have

$$
\begin{gathered}
A^{3 / 2}\left(R^{3}-3 R R_{I}^{2}\right)+E(R-1)=0 \\
A^{3 / 2}\left(3 R^{2} R_{I}-R_{I}^{3}\right)+E R_{I}=0
\end{gathered}
$$

Comparing (58) \& (59), we get,

$$
\frac{R^{3}-3 R R_{I}^{2}}{3 R^{2} R_{I}-R_{I}^{3}}=\frac{R-1}{R_{I}}, \text { i.e. } \quad R_{I}= \pm R \sqrt{\frac{3-2 R}{1+2 R}}
$$

\section{Quantum Cosmology of Zero Loop}

We consider the Hartle-Hawking path integral for 'no boundary' proposal, by taking the wave function, $\psi\left[\mathrm{g}_{\mathrm{ij}}\left(\mathrm{y}^{\mathrm{k}}\right), \phi^{\mathrm{A}}\left(\mathrm{y}^{\mathrm{k}}\right)\right] \approx \psi_{\mathrm{o}-\operatorname{loop}}\left[\mathrm{g}_{\mathrm{ij}}, \phi^{\mathrm{A}}\right]=\sum_{\text {Some extrema }} \mathrm{e}^{-\mathrm{I}\left[\mathrm{g}_{\mu v}, \phi^{\Omega}\right]}$

Where we summing over a small set of extrema of the Euclidean action I, generally complex classical solutions of the field equations.

The zero-loop approximation gives

$$
\psi\left(\mathrm{a}_{\mathrm{b}}, \phi_{\mathrm{b}}\right) \approx \psi_{0 \text {-loop }}\left(\mathrm{a}_{\mathrm{b}}, \phi_{\mathrm{b}}\right)=\sum_{\text {Some extrema }} \mathrm{e}^{-\mathrm{-I}\left[\mathrm{a}_{\mathrm{b}}, \phi_{\mathrm{b}}\right]}
$$

where $\mathrm{I}\left(\mathrm{a}_{\mathrm{b}}, \phi_{\mathrm{b}}\right)$ is the Euclidean action of a classical solution that is compact and has the $\mathrm{S}^{3}$ geometry and homogeneous scalar field as its one and only boundary.

One boundary FRW-scalar histories have a time parameter ' $t$ ' that can be taken to run from 0 (at a regular 'centre') to 1 (at the boundary), and then to have $\phi=\phi(t)$ and four metric.

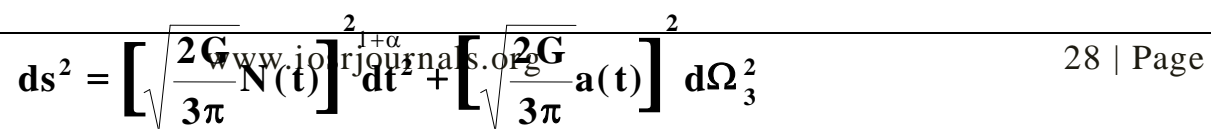


Consider the metric

Where $N(t)$ is the lapse function and $d \Omega_{3}^{2}$ is the metric on a unit 3-sphere $S^{3}$.

If the scalar field potential is the formulas

$\left[\frac{\mathbf{9}}{\mathbf{1 6} \mathbf{G}^{\mathbf{2}}}\right] \mathrm{V}(\phi)$ [with the co-efficient again chosen to simplify below, in terms of the rescaled potential $[\mathrm{V}(\phi)]$, then the Euclidean action of the history is

$\begin{aligned} \mathrm{I} & =-\mathrm{iS}=\int \mathrm{dt}\left(\frac{1}{2 \mathrm{~N}}\left(-\mathrm{aa}^{2}+\mathrm{a}^{3} \dot{\phi}^{2}\right)+\frac{1}{2} \mathrm{~N}\left(-\mathrm{a}+\mathrm{a}^{3} \mathrm{~V}\right)\right. \\ \text { i.e. } \quad \frac{\mathrm{dI}}{\mathrm{dt}} & =0=\frac{\mathbf{1}}{\mathbf{2 N}}\left(-\mathbf{a a}^{2}+\mathbf{a}^{3} \dot{\phi}^{2}\right)+\frac{\mathbf{1}}{\mathbf{2}} \mathbf{N}\left(-\mathbf{a}+\mathbf{a}^{3} \mathbf{V}\right)=\mathbf{L}=\mathbf{H}\end{aligned}$

Here $\phi$ is the scalar field.

\section{X. $\quad$ FRW-Scalar Model with Complex Scale Factor}

We have from the equation (65),

$$
-\dot{\mathrm{a}}^{2}+\mathrm{a}^{2} \dot{\phi}^{2}+\mathrm{N}^{2}\left(-1+\mathrm{a}^{2} \mathrm{~V}\right)=0
$$

We consider $a=R+i R_{I}$ as complex radius. Now substituting ' $a$ ' in the equation (66), and separating real and imaginary part, we have,

and

$$
-\left(\dot{\mathrm{R}}^{2}-\dot{\mathrm{R}}_{1}^{2}\right)+\left(\mathrm{R}^{2}-\mathrm{R}_{1}^{2}\right) \dot{\phi}^{2}-\mathrm{N}^{2} \mathrm{~V}+\mathrm{N}^{2} \mathrm{~V}\left(\mathrm{R}^{2}-\mathrm{R}_{1}^{2}\right)=0
$$

Now we consider,

$$
\begin{gathered}
\dot{\phi}^{2}+\mathrm{N}^{2} \mathrm{~V}-\frac{\dot{\mathrm{R}}}{\mathrm{R}} \frac{\dot{\mathrm{R}}_{1}}{\mathrm{R}_{\mathrm{I}}}=0 \\
\mathrm{H}_{\mathrm{R}}=\frac{\dot{\mathrm{R}}}{\mathrm{R}} \& \mathrm{H}_{\mathrm{I}}=\frac{\dot{\mathrm{R}}_{1}}{\mathrm{R}_{\mathrm{I}}} ; \mathrm{R}(\mathrm{t})=\ell_{\mathrm{p}} \mathrm{e}^{\mathrm{H}_{\mathrm{R}} \mathrm{t}} \text { and } \mathrm{R}_{\mathrm{I}}(\mathrm{t})=\ell_{\mathrm{p}} \mathrm{e}^{\mathrm{H}_{\mathrm{I}} \mathrm{t}}
\end{gathered}
$$

where $\mathrm{R}(0)=\mathrm{R}_{\mathrm{I}}(0)=\ell_{\mathrm{p}}$ and consider $\mathrm{N}(\mathrm{t})=1$.

Thus the equation (68) becomes, $\quad \dot{\phi}^{2}=\mathrm{H}_{\mathrm{R}} \mathrm{H}_{\mathrm{I}}-\mathrm{V}$

\subsection{FRW-Scalar Models with An Exponential Potential V $=\mathrm{e}^{2 \alpha \phi}$}

To illustrate some of these ideas quantitatively, it is helpful to consider the case of an exponential potential

$$
\mathrm{V}(\phi)=\mathrm{e}^{2 \alpha \phi}
$$

where $\alpha$ is a real parameter that characterizes how fast the potential varies as a function of $\phi$.

We have from the equation (69) \& (70), $\quad \frac{\mathrm{d} \phi}{\mathrm{dt}}= \pm \sqrt{\mathrm{H}_{\mathrm{R}} \mathrm{H}_{\mathrm{I}}-\mathrm{e}^{2 \alpha \phi}}$
Integrating, we have

$$
\mathrm{e}^{2 \alpha \phi}\left(\mathrm{A}^{2} \mathrm{e}^{\mp 2 \alpha \sqrt{\mathrm{H}_{\mathrm{R}} \mathrm{H}_{\mathrm{I}}} \mathrm{t}}+1\right)^{2}= \pm 4 \mathrm{H}_{\mathrm{R}} \mathrm{H}_{\mathrm{I}}\left(\mathrm{A}^{2} \mathrm{e}^{\mp 2 \alpha \sqrt{\mathrm{H}_{\mathrm{R}} \mathrm{H}_{\mathrm{I}}} \mathrm{t}}+1\right)-4 \mathrm{H}_{\mathrm{R}} \mathrm{H}_{\mathrm{I}}
$$

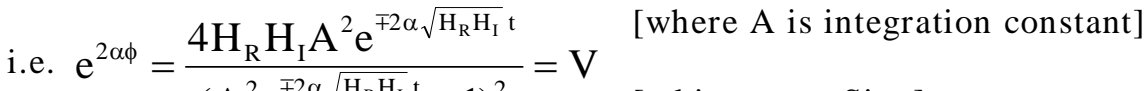

Hence

$$
\left(\mathrm{A}^{2} \mathrm{e}^{\mp 2 \alpha \sqrt{\mathrm{H}_{\mathrm{R}} \mathrm{H}_{\mathrm{I}}} \mathrm{t}}+1\right)^{2} \quad \text { [taking +ve Sign] }
$$

$$
\phi=\frac{1}{\alpha} \log \left(2 \sqrt{\mathrm{H}_{\mathrm{R}} \mathrm{H}_{\mathrm{I}}} \mathrm{A}\right)-\frac{1}{\alpha} \log \mathrm{A}^{2} \mathrm{e}^{\mp \alpha \sqrt{\mathrm{H}_{\mathrm{R}} \mathrm{H}_{\mathrm{I}} \mathrm{t}}}+\mathrm{e}^{ \pm \alpha \sqrt{\mathrm{H}_{\mathrm{R}} \mathrm{H}_{\mathrm{I}} \mathrm{t}}}
$$

Differentiating both side with respect to time, $t$ we get

$$
\dot{\phi}= \pm \sqrt{\mathrm{H}_{\mathrm{R}} \mathrm{H}_{\mathrm{I}}} \frac{\mathrm{A}^{2} \mathrm{e}^{\mp \alpha \sqrt{\mathrm{H}_{\mathrm{R}} \mathrm{H}_{\mathrm{I}}} \mathrm{t}}-\mathrm{e}^{ \pm \alpha \sqrt{\mathrm{H}_{\mathrm{R}} \mathrm{H}_{\mathrm{I}}} \mathrm{t}}}{\mathrm{A}^{2} \mathrm{e}^{\mp \alpha \sqrt{\mathrm{H}_{\mathrm{R}} \mathrm{H}_{\mathrm{I}}} \mathrm{t}}+\mathrm{e}^{ \pm \alpha \sqrt{\mathrm{H}_{\mathrm{R}} \mathrm{H}_{\mathrm{I}}} \mathrm{t}}}
$$

Again, if we take negative sign, then from the equation (71), we have

$$
\begin{aligned}
& \mathrm{V}=\mathrm{e}^{2 \alpha \phi}=\frac{-4 \mathrm{H}_{\mathrm{R}} \mathrm{H}_{\mathrm{I}}\left(\mathrm{A}^{2} \mathrm{e}^{\mp 2 \alpha \sqrt{\mathrm{H}_{\mathrm{R}} \mathrm{H}_{\mathrm{I}}} \mathrm{t}}+2\right)}{\left(\mathrm{A}^{2} \mathrm{e}^{\mp 2 \alpha \sqrt{\mathrm{H}_{\mathrm{R}} \mathrm{H}_{\mathrm{I}}} \mathrm{t}}+1\right)^{2}}
\end{aligned}
$$

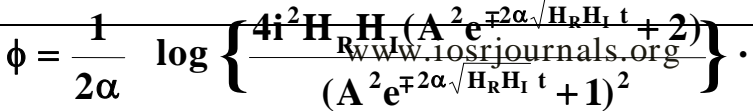


When $\mathrm{A}=1$, then,

$$
\begin{aligned}
& \mathrm{V}=\mathbf{H}_{\mathbf{R}} \mathbf{H}_{\mathrm{I}}\left[\frac{\mathbf{e}^{\mp \alpha \sqrt{\mathrm{H}_{\mathrm{R}} \mathrm{H}_{\mathrm{I}}} \mathrm{t}}+\mathbf{e}^{ \pm \alpha \sqrt{\mathrm{H}_{\mathrm{R}} \mathrm{H}_{\mathrm{I}}}}}{\mathbf{2}}\right]^{-2} \\
& \& \quad \phi=\frac{1}{\alpha} \log \left[\sqrt{\mathbf{H}_{\mathbf{R}} \mathbf{H}_{\mathbf{I}}}\left(\frac{\mathbf{e}^{ \pm \alpha \sqrt{\mathbf{H}_{\mathrm{R}} \mathrm{H}_{\mathbf{I}} \mathrm{t}}}+\mathbf{e}^{\mp \boldsymbol{\alpha} \sqrt{\mathrm{H}_{\mathrm{R}} \mathrm{H}_{\mathbf{I}}}}}{\mathbf{2}}\right)\right]^{-1} \\
& \dot{\phi}= \pm \sqrt{\mathrm{H}_{\mathrm{R}} \mathrm{H}_{\mathrm{I}}} \frac{\mathrm{e}^{\mp \alpha \sqrt{\mathrm{H}_{\mathrm{R}} \mathrm{H}_{\mathrm{I}}} \mathrm{t}}-\mathrm{e}^{ \pm \alpha \sqrt{\mathrm{H}_{\mathrm{R}} \mathrm{H}_{\mathrm{I}}} \mathrm{t}}}{\mathrm{e}^{\mp \alpha \sqrt{\mathrm{H}_{\mathrm{R}} \mathrm{H}_{\mathrm{I}}} \mathrm{t}}+\mathrm{e}^{ \pm \alpha \sqrt{\mathrm{H}_{\mathrm{R}} \mathrm{H}_{\mathrm{I}}} \mathrm{t}}} \\
& \rho \approx \pm \frac{\sqrt{\mathrm{H}_{\mathrm{R}} \mathrm{H}_{\mathrm{I}}}}{2 \mathrm{c}^{2}} \\
& \frac{e^{\mp \alpha \sqrt{\mathrm{H}_{\mathrm{R}} \mathrm{H}_{\mathrm{I}}} t}-\mathrm{e}^{ \pm \alpha \sqrt{\mathrm{H}_{\mathrm{R}} \mathrm{H}_{\mathrm{I}}} \mathrm{t}}}{\frac{2}{\mathrm{e}^{\mp \alpha \sqrt{\mathrm{H}_{\mathrm{R}} \mathrm{H}_{\mathrm{I}}} \mathrm{t}}+\mathrm{e}^{ \pm \alpha \sqrt{\mathrm{H}_{\mathrm{R}} \mathrm{H}_{\mathrm{I}}} \mathrm{t}}}} \\
& 2
\end{aligned}
$$

10.2 The Energy Density of $\phi$-Field

We have from the equation (71)

$$
\phi=\frac{1}{2 \alpha} \log \left\{4 \mathrm{H}_{\mathrm{R}} \mathrm{H}_{\mathrm{I}}\left( \pm \mathrm{A}^{2} \mathrm{e}^{\mp 2 \alpha \sqrt{\mathrm{H}_{\mathrm{R}} \mathrm{H}_{\mathrm{I}}^{\mathrm{t}}}} \pm 1-1\right)-\frac{1}{\alpha} \log \left(\mathrm{A}^{2} \mathrm{e}^{\mp 2 \alpha \sqrt{\mathrm{H}_{\mathrm{R}} \mathrm{H}_{\mathrm{I}}^{\mathrm{t}}}}+1\right)\right.
$$

The inflationary model seems capable of producing the spectrum, through fluctuations in the scalar field $\phi(\mathrm{t})$.

Harrison in 1970 and Zeldovich in 1972 has argued independently, from theoretical considerations that at the time of entering the horizon, the amplitude of a typical density perturbations should have the form $\mathrm{F}(\mathrm{k}) \alpha \mathrm{k}^{-3}$ where
the microwave background

Radiation is $<10^{-5}$, implies that $\left.\mid \delta(\mathrm{k}, \mathrm{t})\right]^{2}<<1$ in the radiation dominated phase of expansion. It can be shown that the root-mean-square fluctuation of mass $M$ as a fraction of average mass contained in a region of size $R$ is proportional to $k^{3}|\delta K|^{2}$ at $k=R^{-1}$. Therefore, for the above $F(k),\left\langle(\delta M / M)^{2}\right\rangle$ will be independent of the scale $\mathrm{R}$ at $\mathrm{t}=\mathrm{t}(\mathrm{k})$, thus giving equal power at all scales at the time they enter the horizon.

A scale-invariant spectrum is indicated by the distribution of discrete large-scale structures.

We write the equation (81), the fluctuation as $F(t)$ over a smoothed average value $\phi_{0}(t)$.

Thus $\quad \phi(\mathrm{t})=\phi_{\mathrm{o}}(\mathrm{t})+\mathrm{F}(\mathrm{t})$

These fluctuations result in fluctuations of energy density.

The energy density of a scalar field is $\quad \rho c^{2} \approx \frac{1}{2} \dot{\phi}^{2}(t)$

The average energy density during inflation being dominated by the constant term $V_{O}$ of the Coleman-Weinberg-potential. We have the density contrast

$$
\delta_{\mathrm{k}}(\mathrm{t})=\frac{\delta \rho \mathrm{c}^{2}}{\mathrm{~V}_{\mathrm{o}}}
$$

We use $\dot{\phi}_{\mathrm{o}}$, the mean evolution of $\phi$ in the slow roll-over phase; but what is $\mathrm{F}(\mathrm{t})$ ? Now in actuality the fluctuations in $\phi$ are of quantum origin but here, in a classical approximation, we are using $F(t)$

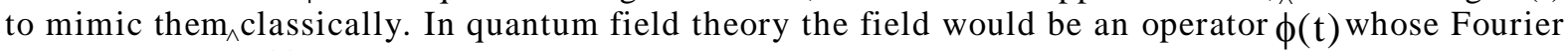
coefficient $\mathrm{q}_{\mathrm{k}}(\mathrm{t})$ are also operators. In a quantum state specified by the wave function $\Psi_{\mathrm{k}}$, the fluctuations of $\hat{q}_{k}(t)$ are given by the dispersion relation $\sigma^{2}(\mathrm{t})=\left\langle\Psi_{\mathrm{k}}\left|\mathrm{q}_{\mathrm{k}}^{2}(\mathrm{t})\right| \Psi_{\mathrm{k}}\right\rangle$

The mean value (in $\mathrm{k}=0$ mode) of $\Psi_{\mathrm{k}}$ being zero. This is because $\phi_{0}$, the average of $\phi$, is homogeneous. Since $\sigma_{k}^{2}(\mathrm{t})$ appears to be a good measure of quantum fluctuations. We may write

$$
\delta_{k}(t)=\frac{\phi_{0}(\mathrm{t})}{\mathrm{V}_{0}} \dot{\sigma}_{\mathrm{k}}(\mathrm{t})
$$


Thus we have taken a semi-classical approximation to estimate the fluctuations in the energy density of the $\phi$ field which act as the seed fluctuation of density during the inflationary phase $t_{i}<t$ $<\mathrm{t}_{\mathrm{f}}$.

\section{Excess production of $\mathbf{J}_{\mathbf{k}}$ bosons in the early universe:}

Let us denote the mass of the $\mathrm{J}$-bosons by $\mathrm{m}_{\mathrm{J}}$, and its coupling strength by $\alpha_{\mathrm{J}}$. The coupling strength depending on what type of particle $J$ is, let us denote by $\Gamma_{\mathbf{c}}$ the rate of collisions that do not conserve the number of $\mathbf{J}_{\mathrm{k}}$ bosons, i.e. collisions in which the $\mathbf{J}$-boson is involved. Denote the characteristic decay rate of the J-boson by $\Gamma_{\mathrm{J}}$, we thus have three time scales to play with: $\Gamma_{\mathrm{J}}^{-1}, \Gamma_{\mathrm{e}^{\prime}}^{-1}$ and $\mathrm{H}_{\mathrm{I}}^{-1}$

At the earliest epochs, with constant temperature $>10^{19} \mathrm{GeV}$, the latent energy was the strongest force between the various constituents of the universe. Other interactions were unimportant under the hypothesis of asymptotic freedom. As the universe continued to changing phase and its constant temperature dropped there was a phase when gravity as well as latent force become weaker while the other interactions still remained unimportant. Thus for $\mathrm{T} \leq 10^{19} \mathrm{GeV}$, the particles remained essentially free for some time.

During this phase it becomes necessary to examine the nature of distribution, functions are as follows. Assuming ideal gas approximation and thermodynamic equilibrium, it is then possible to write down the distribution functions of any given species of particles. Let us use the symbol L to denote typical species $(L=1,2, \ldots)$. Thus $\mathrm{n}_{\mathrm{L}}(\mathrm{P})$ dp denotes the number density of species in the momentum range $(\mathrm{P}, \mathrm{P}+\mathrm{dP})$, where

$$
\mathbf{n}_{L}(\mathbf{P})=\frac{g_{L}}{2 \pi^{2} \hbar^{3}} P^{2}\left[\operatorname{EXP} \frac{E_{L}(P)-\square_{L}}{k T}-1\right]^{-1}
$$

Where $\mathrm{T}=$ the temperature of the distribution, $\mathrm{g}_{\mathrm{L}}=$ the number of spin states of the species, $\mathrm{k}=$ the Boltzmann constant and $\quad \mathbf{E}_{\mathrm{L}}^{2}=\mathbf{c}^{2} \mathbf{P}^{2}+\mathbf{m}_{\mathrm{L}}^{2} \mathbf{c}^{\iota^{2}}$ is the energy corresponding to rest mass $\mathrm{m}_{\mathrm{L}}$ of a typical particle. The quantity $\mu_{\mathrm{L}}$ is the chemical potential of the species $\mathrm{L}$. We set $\mu_{\mathrm{L}}=0, \mathrm{~g}_{\mathrm{L}}=1, \mathrm{~m}_{\mathrm{L}}$ $=0$, for $\mathrm{J}_{\mathrm{k}}$ bosons. Since particles and antiparticles annihilate in pairs and produce $\mathrm{J}_{\mathrm{k}}$ bosons their chemical potentials are equal and opposite. Again we saw that for $\mathrm{T} \leq \mathrm{T}_{\mathrm{J}}$, the distribution function cannot preserve its form under changing phase. Thus it may get distorted from its equilibrium form. Now of the various species in the very early universe, the J-bosons are probably the most massive. Thus, provided they have a high enough value $\mathrm{T}_{\mathrm{J}}$, there is a chance that the $\mathrm{J}$ bosons will first dropout of equilibrium. For this to happen, however, it is also necessary that they have not all decayed by then. The collision rate $\Gamma_{\mathrm{c}^{\prime}} \approx \alpha_{\mathrm{J}}<<\Gamma_{\mathrm{J}}$. A comparison of the three rats shows that $\Gamma_{\mathrm{c}^{\prime}}<\Gamma_{\mathrm{J}}<\mathrm{H}_{\mathrm{I}}$ Soon after gravity became weak that means the amount of equivalent energy is not adequate then the changing phase of the universe with the essentially no interaction between the species.

\section{Concluding Remarks:}

First we studied the unification of the energy group SU(5) with a group SU(6) (In mathematics, the special unitary group of degree $n$ may be denoted by $S U(n)$, which is the group of $n \times n$ unitary matrices with determinant 1 . The group operation is that of matrix multiplication. Again, the special unitary group is a subgroup of the unitary group $U(n)$, consisting of all $n \times n$ unitary matrices, which is itself a sub group of the general linear group $\mathrm{GL}(\mathrm{n}, \mathrm{C})$, where $\mathrm{C}$ is the classical Lie group. The group SU(n) may find wide application in the standard model of physics, particularly SU(2) in the electro-weak interaction and SU(3) in quantum-chromodynamics as well as there introduces a new type energy sources $\mathrm{SU}(6)$ in the present dissertation.) to form a special unitary group SU(11). In these early epochs the dominant form of energy particles moving relativistically. Such particles were mostly moving freely and would collide, of course, but these instances are assumed to have occupied very brief time spans and there effects on motions may be otherwise neglected. The collisions and scattering of the particles would, however, have helped to redistribute their energies and momenta. If these redistribution occurred frequently enough then the system of particles as a whole would have reached a state of thermodynamic equilibrium. In this case, for each species of particles there is a definite rule governing the number of particles in a given range of momentum. 
However, if the large percent of the matter sources in the universe would be of dark-energy type (as the present observations strongly recommend) then one may keep the results here even in the presence of other matter source, keeping in mind that the relevant contribution to the total matter source of the universe is the dark energy. It is shown, at the early epoch, the pressure free ( $\mathrm{p}^{\prime}$ $=\mathrm{p}=0$ ) wider universe and Einstein's universe are identical. It is found that the existence of discrete structure in the universe, ranging from galaxies to super-clusters.

So the space-time of the universe is actually a complex space-time and there is neither any starting point nor any ending point of the wider (measurable in quantum cosmology) universe. Only there exists the initial and ending conditions for narrower (measurable in classically) universe, which emerged from wider universe by the process of changing phase, which is a continuous process. As ocean has close with tide, similarly the wider universe has the same with narrower universe.

Also I think, the existence of new particles confirms at CERN's laboratory declared on $4^{\text {th }}$ July 2012 may belongs to the energy group SU(6).

We showed that in Chaplygin gas scenario, we may observe a positive effective cosmological constant at the late times despite having a negative bare cosmological constant. This is in agreement with the assumption of the existence of the negative cosmological constant in the early universe.

We have constructed wave packets by the appropriate linear combination of the eigenfunctions. The time evolution of the expectation value of the scale factors has been determined in the spirit of the many-worlds interpretation of quantum cosmology. The scale factors considered here never tend to the singular point, i.e. these models may not have singularities at the quantum level.

In the paper, we consider a path integral over all compact Euclidean 4-dimensional geometrics and matter fields by considering a complex scale factor. There were a stage of SUT of Phase transition before GUT. We think field created by the latent energy group. We consider a scalar $\phi$-field. The fluctuation of the scalar field $\phi$ result in fluctuations of energy density.

We find the value of the scale factor, when potential V $=1$ and also the scale factor, related with imaginary time. It has been showed that the energy density changes exponentially with time. We found the time, when $\phi=0$, in terms of the parameter $\mathrm{H}_{\mathrm{R}} \& \mathrm{H}_{\mathrm{I}}$.

\section{Acknowledgement:}

The author is thankful to Prof. Subenoy Chakraborty, Department of Mathematics, Jadavpur University, Kolkata, West-Bengal, India for helpful discussion.

\section{References:}

[1] N. K. Bhadra, The Complex Model of the Universe. IOSRJM. ISSN: 2278-5728; 2; 4 ,Sep. 2012 PP 41-45;

[2] P. J. E. Peebles, and B. Ratra, Astrophy. J. Lett. 325 . L17 (1988) : A. R. G. Vishwakarma, Class. Quant. Grav. 14. 945(1997). .F. Hoyle. G. Burbidge and J. V. Narlikar, Mon. R. Astron. Soc. 286. 173(1997).

[3] T. A. Appelquist, A. Chodos and P. G. O. Freund, Modern Kaluza-Klein Theories, Frontiers in Physics Series, (Volume 65), 1986 (Ed. Addison Wesely). Brian Greene, The Elegant Universe, W.W.Norton\&Company, New York (1999), pp. 357 - 358. A. G. Riess et al. Astrophys. J. 560, 49(2001).

[4] F. Hoyle \& J. V. Narlikar, A new theory of gravitation. Proc. R. Soc., A282, 191 (1964).F. Hoyle \& J. V. Narlikar, A conformal theory of gravitation. Proc. Roy. Soc., A294, 138 (1996).

[5] A. Einstein \& W. deSitter, on the relation between the expansion and the mean density of the universe. Proc. Natl. Acad. Sci. (USA), 18, 213(1932).

[6] Daniel Kleppner, The Gem of General Relativity, Physics Today, April (1993), 9-11.David J. Gross, Can We Scale the Planck Scale? Physics Today June, 9-11 (1989). Stephen W. Hawking, a Brief History of Time, Bantam Books, New York (1988), pp. $134-137$.

[7] Alan Guth and Paul Steinhardt, "The Inflationary Universe" and article in S. W. Hawking, Phys. Lett. 134B, 403 (1984); E. Baum, ibid. 133B, 1885 (1983); .Albert Einstein, Ideas and Opinions, Crown Publishers, New York (1987), PP. 348.

[8] Chaplygin, S. Sci. Mem. Moscow University Mata Phys. 21. 1(1904). P. J. E. Peeblesand B, Ratra, Astrophys. J. Lett. 325. L17 (1988) : A and R. G. Vishwakar-ma, Class, Quant. Grav. 14.945(1997) : F. Hoyle. G. Burbidge and J. V. Narlikar, Mon. R. Astron. Soc. 286. 173(1997).

[9] B. F. Schutz, Phys. Rev. D 2, 2762(1970); M. I. Kalinin, V. N. Melnikov, Grav. Cosmol. 9, 227(2003). Bnnet, C. L. et al. Astrophys. J. Suppl 148 1(2003) arXiv: astro-ph/0302207. Spergel, D. B.et al. : Astrophys. J. Suppl. 148 175(2003), arXiv:astro-ph/0302209.

[10] Lyons, G. W. (1992). Complex solutions for the scalar field model of the universe, Phys. Rev. D46, 1546 - 1550. Alan H. Guth, The Inflationary Universe, Addision-Wesley Co., Inc., Reading, Massachusetts (1997).

[11] Hawking, S. W. and Luttrell, J. C. (1984). The isotropy of the universe, Phys. Lett. B143, 83 - 86.Gibbons, G. W. Hawking, S. W. and Perry, M. J. (1978). Path integrals and the indefiniteness of the gravitational action, Nucl, Phys. B138, $141-154$.

[12] A Linde, Scalar field fluctuations in the expanding universe and the new inflationary scenario. Phys. Lett., B116, 335(1982).S. W. Hawking, Black hole explosions? Nature, 248, 30, (1974). 
[13] E. R. Harrison, fluctuations at the threshold of classical cosmology. Phys. Rev. B1, 2726(1970).Y. B. Zeldovich, A hypothesis, unifying the structure and the entropy of the universe, MNRAS, 160, 1P (1972).

[14] Hawking, S. W. and Hartle, J. B. (1983). Wave function of the universe, Phys. Rev. D28, 2960 - 2975.Hawking, S. W. (1985). The origin of structure in the universe, Phys, Rev. D31, 1777-1791. Hawking, S. W. (1985). The arrow of time in cosmology, Phys. Rev. D32, $2489-2495$. 